\title{
Cell Therapy for Diabetic Retinopathy - A Work in Progress
}

\author{
David Kent
}

Consultant Ophthalmic Surgeon, The Vision Clinic, Kilkenny, Ireland

\begin{abstract}
As the incidence of diabetes continues to rise worldwide, the challenge in preventing vision loss secondary to diabetic retinopathy (DR) remains a formidable one. Current treatments are only indicated in advanced disease when vision loss is imminent or has already occurred. In recent years, due to the discovery that postnatal vasculogenesis plays a role in vascular repair, there is increasing optimism that cell therapy using endothelial progenitor cells (EPCS) can be harnessed therapeutically for conditions such as DR. Although autologous EPC therapy offers promise for DR, EPCs from patients with diabetes are themselves dysfunctional while the diabetic milieu itself contributes further to this dysfunctionality. Additional research is also required to unravel the complete science of vasculogenesis and the role of EPCs in repair so that treatment can be optimised in terms of actual cell choice, pre-conditioning prior to transplantation, maximising cell survival in the recipient and preparation of the recipient tissue to ensure an adequate therapeutic response.
\end{abstract}

\section{Keywords}

Diabetic retinopathy, cell therapy, endothelial progenitor cell, angiogenesis, vasculogenesis, neovascularisation, therapeutic angiogenesis, vascular repair

Disclosure: The author has no conflicts of interest to declare.

Received: 1 October 2011 Accepted: 1 February 2012 Citation: European Ophthalmic Review, 2012;6(2):125-9 DOI: 10.17925/EOR.2012.06.02.125

Correspondence: David Kent, The Vision Clinic, 7 Circular Road, Kilkenny, Ireland. E: dkent@liverpool.ac.uk

In the developed world diabetic retinopathy (DR) is the leading cause of vision loss in the working population. ${ }^{1}$ Coupled with the fact that the prevalence of DR is expected to double by 2025 , the impact on quality of life of affected individuals and the societal cost in general cannot be overstated. ${ }^{2}$ The increasing burden on ophthalmic departments is similarly enormous so the search for novel therapies has become imperative. Laser photocoagulation for DR has been the therapeutic gold standard for over a quarter of a century, while recently we have seen the introduction of intravitreally administered anti-angiogenic therapy following the results of recently conducted clinical trials..$^{3-8}$ Moreover, the recognition of the role of inflammation in DR has also focused the spotlight on the use of intravitreal steroids. ${ }^{9-11}$ These therapies, though variously effective, are late stage interventions that are administered when the disease may have been present for many years and when the risk of vision loss is imminent or may already have occurred. Thus, in the absence of a cure for diabetes, a treatment that could be delivered at an earlier stage when the risk of vision loss is still remote, which is safe and directly addresses the underlying pathology of the disease would be a giant step forward. As cell therapy satisfies these criteria it has potential as a future treatment for DR.

\section{Terminology and Definitions}

The terminology can be confusing when it comes to defining the various stem cell types. In the main, this article concentrates on what we currently understand to be endothelial progenitor cells (EPCS). These cells are derived from the bone marrow and were first isolated by Asahara et al. ${ }^{12}$ We now know that when we use this term we are probably not referring to a single cell entity but instead are referring to a group of cells that are capable of differentiating down an endothelial cell route or lineage..$^{13}$ First, EPCs can be derived from haemangioblasts and express various cell markers including vascular endothelial growth factor receptor 2 (VEGFR-2), CD133 and CD34. ${ }^{14}$ Second, EPCS are considered a subset of cells derived from bone marrow multipotent adult progenitor cells (MAPCS). While these also express VEGFR-2 and CD133, they lack CD34. ${ }^{13}$ Finally, bone marrow-derived myelocytic/monocytic cells can also differentiate into EPCs, express CD14 and form mature endothelial cells that express von Willebrand factor, VEGFR-2 and CD45 in culture. ${ }^{14}$ All EPCs, regardless of their origin, take up acetylated low-density lipoprotein (LDL) and bind Ulex europaeus lectin 1 (UEA 1). ${ }^{1516}$ Thus in vivo at least three groups of 'EPCS' can give rise to mature endothelial cells: haemangioblasts, MAPCs and myelocytic/monocytic cells. ${ }^{14}$

In vitro EPCs can be isolated using cell sorting, by exploiting immunotypic cell surface proteins, or by isolation of the mononuclear fraction of blood followed by cell culture using different substrates and media. Isolation of EPCs using cell sorting is challenging mainly because there is still ambiguity as to what specific surface markers precisely define an EPC, because several markers can overlap between actual progenitors and subsequent cellular differentiation to cells other than EPCS and the practical challenges involved in isolating the small numbers of relevant cells circulating in the peripheral blood..$^{17}$ All these factors can give rise to heterogeneity of the resultant populations. ${ }^{18-20}$ Isolation of the mononuclear fraction of blood and their subsequent culture on various substrates and/or media permits more cellular homogeneity. Using this technique at least two types of EPCs have been identified in adult blood and umbilical cord blood. 21,22 Early EPCs or circulating angiogenic cells are spindle shaped, found within one week of culture, take up acetylated LDL, bind UEA1 and stain for VEGFR-2. Late EPCs or outgrowth endothelial cells can be isolated from umbilical 
cord blood and adult peripheral blood, although those from cord blood have greater proliferation rates and form vascular networks that remain stable for longer than those from adult blood. ${ }^{23,24}$

The foregoing demonstrates ambiguities and controversies surrounding the definition of an actual EPC, mainly due to the different isolation methods. However, regardless of whatever satisfies the precise definition of an EPC, there is fairly unanimous agreement that the unique properties of 'EPCs' should allow them to be exploited as a potential therapeutic vasoreparitive tool.

\section{Neovascularisation - Angiogenesis Versus Vasculogenesis}

Angiogenesis refers to the formation of new blood vessels from the pre-existing vasculature. It occurs by at least two mechanisms. ${ }^{25}$ Endothelial cells within the vasculature migrate and proliferate and then it is the fusion of vacuoles within these cells which form the vascular lumen. This is called sprouting angiogenesis and relies on the generation of hypoxic stimuli and the release of pro-angiogenic factors and proteases that result in breakdown of the basement membrane to permit the migration and proliferation of the endothelial cells into this newly established permissive matrix. However, non-sprouting angiogenesis is poorly understood and occurs when a pre-existing blood vessel splits into two.

Vasculogenesis, defined as the de novo generation of endothelial cells from vascular precursor cells (EPCS) that gives rise to a nascent vasculature, is long recognised as a mechanism for vascular formation in the developing embryo. However, in recent years it has been established that such a mechanism exists postnatally and is thus referred to as postnatal vasculogenesis. ${ }^{16}$ EPCs appear to play at least two roles in this vasculogenic process. First, they can be engrafted directly into the developing vasculature. Second, they can release paracrine or autocrine factors that optimise the local environment to enhance the process of vascular repair. ${ }^{26}$

Neovascularisation, embracing both angiogenesis and vasculogenesis, plays a pivotal role throughout life in both health and disease. In health, physiological neovascularisation maintains vascular integrity. It is also essential for normal wound healing and tissue repair. In disease, the whole neovascular mechanism can be hijacked for tumour growth, survival and metastases. ${ }^{27,28} \mathrm{DR}$, particularly its neovascular form, can be considered a form of tissue repair, albeit with a potentially pathological outcome. If we can accept such a hypothesis, it provides a logical starting point for understanding DR, the role played by neovascularisation and, by implication, postnatal vasculogenesis, and how this vasculogenic component may be harnessed or exploited therapeutically to favourably modify disease outcome. We call this approach cell therapy.

Overall cell therapy and its application can be considered as a novel approach to vascular repair. Indeed, vascular repair itself is but one component of an overall wound or tissue repair process. ${ }^{29}$ Thus, repair of blood vessels using cell therapy cannot be considered in isolation but instead its role must be interpreted in the overall context of tissue repair. ${ }^{30}$ In general terms, repair is what is undertaken by the human body to ensure its survival. An insult to the body such as trauma, infection or the acquisition of a disease activates this reparative mechanism. Indeed, in many instances it is the reparative response to the insult that defines disease morbidity. For example, the entity choroidal neovascularisation
(CNV) satisfies all the histological criteria of a tissue repair response and therefore can more correctly be termed submacular repair. ${ }^{31,32}$ Thus, an alternative aim of therapy for CNV could be to modify this healing response to preserve photoreceptor function and therefore vision. A similar approach using cell therapy can be considered with DR.

\section{Diabetic Retinopathy}

Clinically, we recognise DR by the constellation of intra-retinal haemorrhages, retinal oedema and exudates, cotton wool spots and pre-retinal neovascularisation in the presence of underlying diabetes mellitus. Overall, these clinical changes are graded depending on their severity and this provides important information in regard to the risk of future vision loss and by extension the optimum time to intervene with laser therapy with the aim of preventing this loss of vision. ${ }^{3-6}$ In broad terms we can classify DR into non-proliferative or proliferative. Vision loss in DR is most commonly due to macular oedema but can also occur secondary to bleeding from pre-retinal neovascularisation or in advanced disease tractional retinal detachment. We can further define the extent of the disease by using fluorescein angiography and ocular coherence tomography, but to really understand DR we must delve into the retina at a cellular level. This demonstrates that chronic hyperglycaemia causes dysfunction of the neurovascular unit and breakdown of the inner blood retinal barrier (BRB) due to injury and eventual death of key cells such as pericytes, vascular endothelial cells and Mueller cells. ${ }^{33}$ Initially, inner BRB breakdown causes retinal oedema and when it involves the macula, vision loss can ensue. Further progression and cell loss leads to acellular or hypocellular capillaries and ischaemia, a process often referred to as progressive vasodegeneration. ${ }^{1,34}$

\section{The Therapeutic Challenge of Diabetic Retinopathy}

At its most fundamental level, DR is a disease at the capillary level, a microangiopathy or even an endotheliopathy where progressive disease eventually gives rise to retinal ischaemia., ${ }^{11,34-36}$ The therapeutic challenge is quite straightforward in theory - neutralisation of ischaemia and preservation of vision. Our approach to ischaemic DR is the destruction of these ischaemic/hypoxic areas of retina with laser, thus removing the hypoxic stimulus and the pro-angiogenic tide that this promotes. ${ }^{1,37}$ Although by modern terms this may seem like quite a blunt instrument, it has its origins in the 1950s-1970s through anecdotal observations and the clinical trials that these first observations spawned that such an approach prevented blindness, an astonishing breakthrough by any standards. ${ }^{3-6,38,39}$ These results, together with the macular photocoagulation trials for age-related macular degeneration, tailored our whole approach to the modern management of the acquired vascular retinopathies, including DR, that of anti-angiogenesis. ${ }^{40-42}$ In general, our most up-to-date DR therapy combines laser, essentially thermo-destructive anti-angiogenesis, with anti-angiogenesis at the molecular level, through the inhibition of VEGF. ${ }^{7.9}$ Of course this approach works but at a pathobiological level it is a late stage intervention. Over the past 10-15 years with an increased understanding of neovascularisation, both physiological and pathological, there is now the realisation that it may be possible to intervene earlier and for the first time consider 'fixing' damaged blood vessels rather than collaborating with the disease itself to cause their destruction. In short we must at least begin to embrace the concept of therapeutic angiogenesis rather than the current anti-angiogenic approach. ${ }^{35}$ The challenges that lie ahead in advocating and realising therapeutic angiogenesis are practical and ideological. 
DR is a disease at the capillary level so it is not amenable to approaches that can be used for large vessel disease such as vascular bypass or stenting, techniques routinely used by our vascular colleagues. Our approach therefore must by necessity be pitched at the cellular or molecular level to repair damaged but functioning capillaries or revascularise acellular non-functioning capillaries.

\section{Endothelial Progenitor Cells from Patients with Diabetes Contribute to Retinopathy}

Based on its success in several clinical trials, cell therapy should be a significant step forwards in how we deal with the risk of vision loss associated with DR. ${ }^{43,44}$ However, there are important issues to consider in relation to diabetes before optimising our therapeutic approach. In simple terms we can consider DR occurring as a result of a mismatch between the rate of endothelial cell loss due to disease and the inability of EPCs to replace these cells. Thus it appears that diabetes can impair the vasoreparitive ability of EPCs. ${ }^{45}$ The evidence suggests that this impairment of EPC function is occurring on many levels on the journey from the bone marrow to the retina. First, the peripheral neuropathy associated with diabetes causes dysfunction of the circadian release of EPCs. ${ }^{46}$ Second, underlying peripheral vascular disease can similarly cause decreased levels of circulating EPCs. ${ }^{47}$ Third, even after mobilisation from the bone marrow, migration to the site of injury appears dysfunctional due to EPC intracellular defects in nitric oxide metabolism. ${ }^{48}$ Importantly it appears that this reduced nitric oxide bioavailability can be corrected pharmacologically. ${ }^{49}$

Transforming growth factor $\beta$ (TGF- $\beta$ ) is known to regulate the balance of multiple EPC functions such as proliferation, differentiation and quiescence. ${ }^{50,51}$ However the serum levels and perhaps even the intracellular levels of TGF- $\beta$ in EPCs are elevated in diabetes and it has been proposed that this could be one of the mechanisms whereby chronic hyperglycaemia causes cellular injury by promoting cellular senescence and growth arrest. ${ }^{52.53}$ In general then, it begs the question, does elevated TGF- $\beta$ in diabetes contribute to EPC dysfunctionality, but also can these levels be reduced to more physiological levels and will this permit the return to EPC functional normality and consequently normal vascular repair in the retina? Bhatwadekar et al. transiently inhibited endogenous TGF- $\beta$ in peripheral blood CD34+ cells by treating them ex vivo with phosphorodiamidate morpholino oligomers and tested cell function in vitro and in two in vivo experimental models of vascular injury - the acute ischaemia-reperfusion retina model and laser to Bruch's membrane model. ${ }^{54-56}$ The results were striking in terms of correction of diabetic EPC dysfunctionality. Essentially, EPC survival, proliferation, migration, engraftment and homing to the site of injury were all markedly enhanced compared with controls. It appears that transient inhibition of TGF- $\beta$ leads to increased surface expression of CXCR4 with activation of these receptors with stromal-derived factor-1 (SDF-1) leading to increased nitric oxide production. This in turn enhances EPC migration and this combined with increased EPC proliferation and survival generates a robust vascular reparative response..$^{54}$

\section{Feasibility of Reparative Vasculogenesis}

The above observations raise the possibility that patients can undergo autologous EPC transfusion once the dysfunctionality can be corrected ex vivo. This could also permit the pre-conditioning of cells with other growth factors, genetic modification, pharmacological manipulation or subjecting them to physical treatments such as hypoxia. ${ }^{57-60}$ Even allowing for correctional strategies and pre-conditioning there are still many other practical issues to consider. In reality diabetes, once diagnosed, is a chronic disease lasting the lifetime of the patient, has a variable course from patient to patient and can be present with other related and unrelated co-morbidities. Cell therapy in such varying clinical scenarios may at the very least give hugely variable results. This certainly has been the experience when cell therapy has been subject to clinical trials in non-ophthalmic settings. ${ }^{43,61-64}$ There is also the issue of optimising the most practical route of administration of cells, timing of administration, the likely need for re-treatments, how treatments will affect other aspects of diabetes and of course complications associated with the treatment itself and the route of administration, not to mention how this may affect the bone marrow stem cell niche in the long term. In addition the fate of the individual cells has to be considered. Clearly further research is required to unravel these important therapeutic issues. ${ }^{65,66}$

Factors limiting the efficacy of cell therapy include loss of homing receptors that may result from pre-conditioning ex vivo and massive loss of cells on the journey to or upon arrival in the potentially hostile environment of the diseased retina. Despite the fact that these practical obstacles can be overcome, how will the EPC itself perform in an environment that is potentially more hostile than the optimum conditions encountered in the experimental models? In general, many of these models are models of acute injury and therefore the conditions present are ideal to study the effects of a potential therapy in modifying 'disease' outcome. However, diabetes, often in conjunction with dyslipidaemia and hypertension, is characterised by chronic hyperglycaemia that has multiple adverse consequences for biological systems in the body, including the development of DR. ${ }^{46,47}$ Even DR most likely arises from several distinct but overlapping molecular pathways that give rise to the picture we recognise clinically. This setting is far from the ideal testing ground in experimental models and in itself may pose an interesting therapeutic challenge in terms of whether reparative vasculogenesis will work in the diseased retinal habitat and whether this habitat can be favourably modified so that it becomes more conducive to cell therapy. For example, the most likely candidate for enhancing EPC recruitment to the retina could be the manipulation of chemokine SDF-1 and/or its receptor CXCR4. We know it is significantly up-regulated in various experimental models of retinal disease and in patients with vascular retinopathies. ${ }^{67-70}$ Several reports testify to the benefit of SDF-1-mediated mobilisation of bone-marrow-derived stem cells and their role in modifying experimental myocardial infarction through enhanced angiogenesis and improved cardiac function. ${ }^{71-74}$ SDF-1 also appears to have other pertinent roles that add to its attraction as a therapeutic candidate to enhance stem cell behaviour. These include activation of cell survival signalling and cytoprotective pathways during repair. ${ }^{75,76}$ Ultimately, because DR is progressive it raises the possibility that more advanced retinopathy may even be more refractory to intervention. The solution may therefore be to intervene at an early stage. Currently we intervene only when complications have arisen (diabetic macular oedema) or when complications and their visual sequelae are likely (proliferative diabetic retinopathy). As we have seen, this process is based on clinical grading. Earlier intervention could render such an approach redundant and if so we will require a new clinical or molecular signposting system to guide clinicians.

With respect to the mode of delivery, the last number of years has seen intravitreal injections become the standard method for delivering drugs 
to the retina. As this method is safe and easy to perform it would seem logical to use it to administer stems cells for retinal disease. Certainly, in animal models, EPCS have been shown to home to the retina when delivered to the vitreous by injection. ${ }^{48,54}$

\section{Conclusion}

In summary, DR remains a significant and increasing cause of visual morbidity throughout the world. Current therapies, though effective, are delivered only when advanced disease is present and do not address the underlying pathobiology. Developments in cell therapy have now reached a stage whereby it can be considered as a potential therapy for DR. Moreover, cell therapy can be delivered at an early stage of the disease before vision loss occurs or is likely to occur. Realistically though many obstacles still need to be overcome before it becomes a therapy that is both practical and reproducible. First, there are still knowledge gaps concerning the EPCs themselves, including what actually constitutes an EPC. Second, we need to understand more about how best to pre-condition EPCs prior to transplantation. Third, we need to understand more about the fate of EPCS once they are within the recipient and that adequate numbers reach the required treatment bed. Fourth, we need to understand more about the dysfunctionality of EPCs in patients with diabetes and how the diabetic environment itself needs to be modified to optimise EPC performance. Finally, we need to create the optimal permissive environment within the treatment field that permits EPCS to function correctly. This requires a greater understanding of both the cellular- and cytokine-driven processes that constitute DR. Only with this additional knowledge can cell therapy be realised in the future.
1. Fong DS, Aiello LP, Ferris FL, 3rd, Klein R, Diabetic retinopathy, Diabetes Care, 2004;27:2540-53.

2. Candrilli SD, Davis KL, Kan HJ, et al., Prevalence and the associated burden of illness of symptoms of diabetic peripheral neuropathy and diabetic retinopathy, J Diabetes Complications, 2007;21:306-14.

3. Preliminary report on effects of photocoagulation therapy The Diabetic Retinopathy Study Research Group, Am J Ophthalmol, 1976;81:383-96.

4. Photocoagulation for diabetic macular edema. Early Treatment Diabetic Retinopathy Study Report no. 1 Early Treatment Diabetic Retinopathy Study research group Arch Ophthalmol, 1985;103:1796-806.

5. Indications for photocoagulation treatment of diabetic retinopathy: Diabetic Retinopathy Study Report no. 14. The Diabetic Retinopathy Study Research Group, Int Ophthalmol Clin, 1987;27:239-53.

6. Early photocoagulation for diabetic retinopathy. ETDRS report number 9. Early Treatment Diabetic Retinopathy Study Research Group, Ophthalmology, 1991;98:766-85. Elman MJ, Aiello LP, Beck RW, et al., Randomized trial evaluating ranibizumab plus prompt or deferred laser or triamcinolone plus prompt laser for diabetic macular edema, Ophthalmology, 2010;117:1064-77.

8. Massin P, Bandello F, Garweg JG, et al., Safety and efficacy of ranibizumab in diabetic macular edema (RESOLVE Study): a 12-month, randomized, controlled, double-masked, multicenter phase II study, Diabetes Care, 2010;33:2399-405

9. Googe J, Brucker AJ, Bressler NM, et al., Randomized trial evaluating short-term effects of intravitreal ranibizumab or triamcinolone acetonide on macular edema after focal/grid laser for diabetic macular edema in eyes also receiving laser for diabetic macular edema in eyes also receiving

panretinal photocoagulation, Retina, 2011;31:1009-27. disease?, Br J Ophthalmol, 2002;86:363-5.

11. Adamis AP, Berman AJ, Immunological mechanisms in the pathogenesis of diabetic retinopathy, Semin Immunopathol, 2008;30:65-84.

12. Asahara T, Murohara T, Sullivan A, et al., Isolation of putative progenitor endothelial cells for angiogenesis, science, 1997:275:964-7.

13. Khakoo AY, Finkel T, Endothelial progenitor cells, Annu Rev Med, 2005;56:79-101.

14. George AL, Bangalore-Prakash P, Rajoria S, et al., Endothelial progenitor cell biology in disease and tissue regeneration, J Hematol Oncol, 2011;4:24.

15. Rehman J, Li J, Orschell CM, March KL, Peripheral blood 'endothelial progenitor cells' are derived from monocyte/macrophages and secrete angiogenic growth factors, circulation, 2003:107:1164-9.

16. Rafii S, Lyden D, Therapeutic stem and progenitor cell transplantation for organ vascularization and regeneration, Nat Med, 2003:9:702-12.

17. Van Craenenbroeck EM, Conraads VM, Van Bockstaele DR, et al., Quantification of circulating endothelial progenitor cells: a methodological comparison of six flow cytometric approaches. I Immunol Methods, 2008;332:31-40.

18. Krenning G, Dankers PY, Jovanovic D, et al., Efficient differentiation of $\mathrm{CD} 14+$ monocytic cells into endothelial cells on degradable biomaterials, Biomaterials, 2007;28:1470-9.

19. Loomans $\mathrm{CJ}$, Wan $\mathrm{H}$, de Crom $\mathrm{R}$, et al., Angiogenic murine endothelial progenitor cells are derived from a myeloid bone marrow fraction and can be identified by endothelial NO marrow fraction and can be identified by endo
synthase expression, Arterioscler Thromb Vasc Biol, 2006:26:1760-7

20. Yoder MC, Defining human endothelial progenitor cells, J Thromb Haemost, 2009; 7(Suppl. 1):49-52.

21. Hur J, Yoon $\mathrm{CH}$, Kim HS, et al., Characterization of two types of endothelial progenitor cells and their different contributions to neovasculogenesis, Arterioscler Thromb Vasc Biol, 2004:24:288-93.

22. Yoder MC, Mead LE, Prater D, et al., Redefining endothelial progenitor cells via clonal analysis and hematopoietic stem/progenitor cell principals, Blood, 2007;109:1801-9.
23. Au P, Daheron LM, Duda DG, et al., Differential in vivo potential of endothelial progenitor cells from human umbilical cord blood and adult peripheral blood to form functional long-lasting vessels, Blood, 2008;111:1302-5.

24. van Beem RT, Verloop RE, Kleijer M, et al., Blood outgrowt endothelial cells from cord blood and peripheral blood: angiogenesis-related characteristics in vitro, J Thromb Haemost, 2009; 7:217-26.

25. Risau W, Mechanisms of angiogenesis, Nature, 1997;386:671-4.

26. Tongers J, Roncalli JG, Losordo DW, Role of endothelia progenitor cells during ischemia-induced vasculogenesis and collateral formation, Microvasc Res, 2010;79:200-6.

27. Asahara T, Masuda H, Takahashi T, et al., Bone marrow origin of endothelial progenitor cells responsible for postnatal vasculogenesis in physiological and pathological neovascularization, Circ Res, 1999;85:221-8.

28. Dong $\mathrm{F}, \mathrm{Ha} X \mathrm{X}$, Effect of endothelial progenitor cells in neovascularization and their application in tumor therapy, Chin Med I (Engl), 2010;123:2454-60.

29. Hunt TK, Basic principles of wound healing, I Trauma 1990;30:S122-8.

30. Tonnesen MG, Feng $X$, Clark RA., Angiogenesis in wound healing, I Investig Dermatol Symp Proc, 2000;5:40-6.

31. Grossniklaus HE, Ling JX, Wallace TM, et al., Macrophage and retinal pigment epithelium expression of angiogenic cytokines in choroidal neovascularization, Mol Vis, 2002;8:119-26

32. Kent D, Sheridan C, Choroidal neovascularization: a wound healing perspective, Mol Vis, 2003;9:747-55.

33. Reichenbach A, Wurm A, Pannicke T, et al., Muller cells as players in retinal degeneration and edema Graefes Arch Clin Exp Ophthalmol, 2007;245:627-36.

34. Hadi HA, Suwaidi JA, Endothelial dysfunction in diabetes mellitus, Vasc Health Risk Manag, 2007;3:853-76.

35. Stitt AW, O'Neill CL, O'Doherty MT, et al., Vascular stem cells and ischaemic retinopathies, Prog Retin Eye Res, 2011;30:149-66

36. Khan ZA, Farhangkhoee $H$, Chakrabarti S, Towards newe molecular targets for chronic diabetic complications, Curr Vasc Pharmacol, 2006:4:45-57.

37. Walshe TE, D'Amore PA, The role of hypoxia in vascular injury and repair, Annu Rev Pathol, 2008;3:615-43.

38. Meyer-Schwickerath G, Licktkoagulation, Buch Augenarzt, 1959:33.

39. Okun $E$, The effectiveness of photocoagulation in the therapy of proliferative diabetic retinopathy (PDR). (A controlled study in 50 patients). Trans Am Acad Ophthalmol Otolaryngol, 1968;72:246-52.

40. Argon laser photocoagulation for senile macular degeneration. Results of a randomized clinical trial Arch Ophthalmol, 1982;100:912-18.

41. Argon laser photocoagulation for neovascular maculopathy Three-year results from randomized clinical trials. Macular Photocoagulation Study Group, Arch Ophthalmol 1986;104:694-701.

42. Krypton laser photocoagulation for neovascular lesions of age-related macular degeneration. Results of a randomized clinical trial. Macular Photocoagulation Study Group, Arch Ophthalmol, 1990;108:816-24.

43. Kawamoto A, Katayama M, Handa N, et al., Intramuscular transplantation of G-CSF-mobilized CD34(+) cells in patients transplantation of G-CSF-mobilized CD34(+) cells in patients
with critical limb ischemia: a phase $1 / /$ lla, multicenter, singlewith critical limb ischemia: a phase I/lla, multice
blinded, dose-escalation clinical trial, stem cells, blinded, dose-escalas;27:2857-64.

44. Sekiguchi $\mathrm{H}$, li M, Losordo DW, The relative potency and safety of endothelial progenitor cells and unselected mononuclear cells for recovery from myocardial infarction and ischemia, I Cell Physiol, 2009;219:235-42.

45. Tepper OM, Galiano RD, Capla JM, et al., Human endothelia progenitor cells from type II diabetics exhibit impaired proliferation, adhesion, and incorporation into vascular structures, circulation, 2002:106:2781-6.

46. Busik JV, Tikhonenko M, Bhatwadekar A, et al., Diabetic retinopathy is associated with bone marrow neuropathy and a depressed peripheral clock, J Exp Med, 2009;206:2897-906

47. Fadini GP, Sartore S, Schiavon M, et al., Diabetes impairs progenitor cell mobilisation after hindlimb ischaemiareperfusion injury in rats, Diabetologia, 2006;49:3075-84

48. Caballero S, Sengupta N, Afzal A, et al., Ischemic vascular damage can be repaired by healthy, but not diabetic, endothelial progenitor cells, Diabetes, 2007:56:960-7.

49. Segal MS, Shah R, Afzal A, et al., Nitric oxide cytoskeletalinduced alterations reverse the endothelial progenitor cell induced alterations reverse the endothelial progenitor
migratory defect associated with diabetes, Diabetes, 2006;55:102-9.

50. Keller JR, Mantel C, Sing GK, et al., Transforming growth factor beta 1 selectively regulates early murine hematopoietic progenitors and inhibits the growth of IL-3dependent myeloid leukemia cell lines, J Exp Med, 1988;168:737-50.

51. Ruscetti FW, Bartelmez SH, Transforming growth factor beta, pleiotropic regulator of hematopoietic stem cells: potential physiological and clinical relevance, Int J Hematol, 2001;74:18-25.

52. Anjaneyulu M, Berent-Spillson $A$, Inoue $\mathrm{T}$, et al., Transforming growth factor-beta induces cellular injury in experimental diabetic neuropathy, Exp Neurol, 2008;211:469-79.

53. Untergasser G, Gander R, Rumpold H, et al., TGF-beta cytokines increase senescence-associated betagalactosidase activity in human prostate basal cells by supporting differentiation processes, but not cellular senescence, Exp Gerontol, 2003;38:1179-88.

54. Bhatwadekar AD, Guerin EP, Jarajapu YP, et al., Transient inhibition of transforming growth factor-beta1 in human diabetic CD34+ cells enhances vascular reparative diabetic CD34+ cells enhances vascur
functions, Diabetes, 2010;59:2010-19.

55. Grossniklaus HE, Kang SJ, Berglin L, Animal models of choroidal and retinal neovascularization, Prog Retin Eye Res, 2010;29:500-19.

56. Tobe T, Ortega S, Luna JD, et al., Targeted disruption of the FGF2 gene does not prevent choroidal neovascularization in a murine model, Am I Pathol, 1998;153:1641-6.

57. Brandt JE, Bartholomew AM, Fortman JD, et al., Ex vivo expansion of autologous bone marrow CD34(+) cells with porcine microvascular endothelial cells results in a graft porcine microvascular endothelial cells results in a grate capable of rescuing

58. Madeddu $P$, Therapeutic angiogenesis and vasculogenesis for tissue regeneration, Exp Physiol, 2005;90:315-26.

59. Noort WA, Feye D, Van Den Akker F, et al., Mesenchymal stromal cells to treat cardiovascular disease: strategies to improve survival and therapeutic results, Panminerva Med, 2010;52:27-40.

60. Pesce M, Burba I, Gambini E, et al., Endothelial and cardiac progenitors: boosting, conditioning and (re)programming for cardiovascular repair, Pharmacol Ther, 2011:129:50-61.

61. Erbs S, Linke A, Schachinger V, et al., Restoration of microvascular function in the infarct-related artery by intracoronary transplantation of bone marrow progenitor cells in patients with acute myocardial infarction: the Doppler Substudy of the Reinfusion of Enriched Progenitor Cells and Infarct Remodeling in Acute Myocardial Infarction (REPAIR-AMI) trial, circulation, 2007;116:366-74.

62. Losordo DW, Schatz RA, White CJ, et al., Intramyocardia transplantation of autologous CD34+ stem cells for intractable angina: a phase 1/lla double-blind, randomized controlled trial, Circulation, 2007:115:3165-72.

63. Lunde K, Solheim S, Aakhus S, et al., Intracoronary injection of mononuclear bone marrow cells in acute myocardial infarction, N Engl I Med, 2006;355:1199-209.

64. Strauer BE, Brehm M, Zeus T, et al., Repair of infarcted myocardium by autologous intracoronary mononuclea bone marrow cell transplantation in humans, Circulation 2002;106:1913-18.

65. Malliaras $\mathrm{K}$, Kreke $\mathrm{M}$, Marban $\mathrm{E}$, The stuttering progress of cell therapy for heart disease, Clin Pharmacol Ther, 
2011;90:532-41

66. Menasche $P$, Cardiac cell therapy: lessons from clinica trials, I Mol Cell Cardiol, 2011;50:258-65.

67. Butler JM, Guthrie SM, Koc M, et al., SDF-1 is both necessary and sufficient to promote proliferative retinopathy, I Clin Invest, 2005;115:86-93.

68. Ki IY, Arimura N, Noda Y, et al., Stromal-derived factorand inflammatory cytokines in retinal vein occlusion, Curr Eye Res, 2007;32:1065-72.

69. Lai P, Li T, Yang J, et al., Upregulation of stromal cellderived factor 1 (SDF-1) expression in microvasculature endothelial cells in retinal ischemia-reperfusion injury, Graefes Arch Clin Exp Ophthalmol, 2008;246:1707-13.
70. Lima e Silva R, Shen J, Hackett SF, et al., The SDF-1/CXCR4 ligand/receptor pair is an important contributor to severa types of ocular neovascularization, FAse J, 2007;21:3219-30

71. Abbott JD, Huang Y, Liu D, et al., Stromal cell-derived factor-1alpha plays a critical role in stem cell recruitment to the heart after myocardial infarction but is no sufficient to induce homing in the absence of injury, Circulation, 2004;110:3300-5.

72. Askari AT, Penn MS, Stromal cell-derived factor-1 mediates stem cell homing and tissue regeneration, Discov Med, 2003:3:46-7

73. Elmadbouh I, Haider H, Jiang S, et al., Ex vivo delivered stromal cell-derived factor-1alpha promotes stem cell homing and induces angiomyogenesis in the infarcted myocardium, J Mol Cell Cardiol, 2007;42:792-803.

74. Kucia M, Dawn B, Hunt G, et al., Cells expressing early cardiac markers reside in the bone marrow and are mobilized into the peripheral blood after myocardial infarction, Circ Res, 2004:95:1191-9.

75. Hu X, Dai S, Wu WJ, et al., Stromal cell derived factor-1 alpha confers protection against myocardial ischemia/reperfusion injury: role of the cardiac stromal cell derived factor-1 alpha CXCR4 axis, Circulation, 2007;116:654-63.

76. Saxena A, Fish JE, White MD, et al., Stromal cell-derived factor-1alpha is cardioprotective after myocardial infarction, Circulation, 2008;117:2224-31. 